\title{
Pivot-and-bond model explains microtubule bundle formation
}

\author{
Marcel Prelogović, ${ }^{1, *}$ Lora Winters,,${ }^{2, *}$ Ana Milas, ${ }^{3, *}$ Iva M. Tolić, ${ }^{2,3, \dagger}$ and Nenad Pavin ${ }^{1, \hbar}$ \\ ${ }^{1}$ Department of Physics, Faculty of Science, University of Zagreb, Bijenička cesta 32, 10000 Zagreb, Croatia \\ ${ }^{2}$ Max Planck Institute of Molecular Cell Biology and Genetics, Pfotenhauerstr. 108, 01307 Dresden, Germany \\ ${ }^{3}$ Division of Molecular Biology, Ruđer Bošković Institute, Bijenička cesta 54, 10000 Zagreb, Croatia
}

(Received 18 April 2017; revised manuscript received 24 April 2019; published 9 July 2019)

\begin{abstract}
During mitosis, microtubules form a spindle, which is responsible for proper segregation of the genetic material. A common structural element in a mitotic spindle is a parallel bundle, consisting of two or more microtubules growing from the same origin and held together by cross-linking proteins. An interesting question is what are the physical principles underlying the formation and stability of such microtubule bundles. Here we show, by introducing the pivot-and-bond model, that random angular movement of microtubules around the spindle pole and forces exerted by cross-linking proteins can explain the formation of microtubule bundles as observed in our experiments. The model predicts that stable parallel bundles can form in the presence of either passive crosslinkers or plus-end directed motors, but not minus-end directed motors. In the cases where bundles form, the time needed for their formation depends mainly on the concentration of cross-linking proteins and the angular diffusion of the microtubule. In conclusion, the angular motion drives the alignment of microtubules, which in turn allows the cross-linking proteins to connect the microtubules into a stable bundle.
\end{abstract}

DOI: $10.1103 /$ PhysRevE.100.012403

\section{INTRODUCTION}

During mitosis the cell forms a spindle, a complex selforganized molecular machine composed of bundles of microtubules (MTs), which segregates the chromosomes into two daughter cells [1]. MTs are thin stiff filaments that typically extend in random directions from two spindle poles [2]. MTs that extend from the same pole can form parallel bundles, whereas MTs originating from opposite spindle poles form antiparallel bundles [3-5]. Stability of MT bundles is ensured by cross-linking proteins, which bind along the MT lattice, connecting neighboring MTs. Cross-linking occurs only if the distance between the MTs is comparable with the size of a cross-linking protein. These proteins can be divided into two classes: (i) proteins that cross-link MTs without directed movement along the MT, such as Ase1/PRC1 [6]; (ii) motor proteins that walk along the MT either toward the plus end of the MT, such as Cut7/Eg5 [7,8], or toward the minus end, such as Ncd $[9,10]$.

Spindle self-organization was studied in different biological systems and several theoretical models were proposed. Formation of antiparallel bundles of MTs in somatic cells of higher eukaryotes was investigated by computer simulations, which include MTs that grow in random directions from two spindle poles and motor proteins that link them [11]. Further, several studies have explored the regulation of the size of the antiparallel overlaps [12-14]. It was shown that in Drosophila embryo molecular motors acting on antiparallel MT bundles and MT dynamics generate forces to separate the spindle

\footnotetext{
*These authors contributed equally to this work.

†tolic@irb.hr.

${ }^{\ddagger}$ npavin@phy.hr
}

poles during prophase [15-17]. In the "slide and cluster" model, which is relevant for spindle formation in Xenopus eggs, MTs are nucleated near chromosomes, transported away from the center and clustered together [18,19]. Applications of the liquid crystal theory show that the spindle organization is regulated on the local level by cross-linking and steric interactions [20,21]. In budding yeast, it was suggested that MTs growing in arbitrary directions from the opposite spindle poles can change their direction due to minus end directed kinesin-14 motors bound to both MTs and get aligned, forming antiparallel bundles [22]. Recent experiments have provided direct observation of antiparallel bundle formation in fission yeast, where MTs meet at an oblique angle and subsequently rotate into antiparallel alignment [23]. Similarly, rotation of astral MTs was implicated in spindle positioning in budding yeast [24]. MT rotational diffusion about a pivot at the spindle pole was included in the models for yeast spindle formation including kinetochore capture [23,25-32], and rotational diffusion was also considered in a model for MT alignment in vitro [33].

In addition to antiparallel MT bundles, MTs can also be organized into parallel bundles [1]. The most prominent parallel bundles are kinetochore fibers ending at the kinetochore. Yet, parallel bundles can form also independently of kinetochores. During meiosis, parallel bundles generate oscillatory movement of the nucleus, which promotes meiotic recombination $[5,34,35]$. When MTs are not properly bundled, nuclear oscillations are impaired [36]. Similarly, parallel bundles are formed in early mitosis, where they can capture kinetochores [26]. However, a physical model for parallel bundle formation, as well as direct observation of this process in vivo, is missing.

In this paper, we develop theory to explore the formation of parallel MT bundles, which is motivated by our experiments. We introduce the pivot-and-bond model for the formation of 
parallel MT bundles, which includes random angular motion of MTs around the spindle pole [23,26], along with the attractive forces exerted by cross-linking proteins. The model predicts faster bundle formation if MTs diffuse faster and the density of cross-linking proteins is higher, which we confirm experimentally. We conclude that the angular motion drives the alignment of MTs, which in turn allows the cross-linking proteins to connect the MTs into a stable bundle.

\section{RESULTS}

\section{A. Experimentally observed bundle formation}

The process of MT bundle formation can be observed experimentally in the fission yeast Schizosaccharomyces pombe because of a small number of MTs in the spindle. At the onset of mitosis, two spindle pole bodies nucleate MTs that form the spindle. To better observe the process of bundle formation, we increased the distance between spindle pole bodies by exposing metaphase cells to cold stress [37]. We observed that once the cold stress is relieved, MTs regrow from the spindle pole bodies and perform angular motion [26]. Some MTs growing from one spindle pole come into contact with an MT growing from the same spindle pole, thereby forming a parallel bundle (Fig. 1(a), Supplemental Material Movie 1 [38]). An increase of the tubulin-GFP signal intensity in the bundle after the contact between the two MTs shows that a stable MT bundle is formed, arguing against the scenario in which one of the MTs depolymerized [Fig. 1(b)]. MTs can also form antiparallel bundles, which was studied in our recent work [26], and MTs growing at an angle with respect to this antiparallel bundle can eventually join it in a manner similar to the parallel bundle formation (Fig. 1(c), Supplemental Material Movie 2 [38]). Such events are also accompanied by an increase in the tubulin-GFP signal intensity in the spindle, suggesting an increase in the number of MTs in the spindle [Fig. 1(d)]. In an independent set of experiments, we used cells with GFP-labeled Mal3, a protein that binds to the growing end of the MT [39], and imaged them at a finer time resolution (Fig. 1(e), Supplemental Material Movie 3 [38]). As with the tubulin-GFP label, we observed bundling events and the accompanied increase in the intensity of GFP in the spindle [Fig. 1(f)]. The bundling process does not depend on kinetochores, based on our previous observation that such bundles are found mainly without kinetochores attached to them (see Additional file 1: Figs. S2e in Ref. [26]). Also, in our experiments, we did not observe unbundling events after the bundles were formed. In all these scenarios of MT bundle formation, MTs performed angular motion around the spindle pole, which allowed them to approach each other and form a bundle.

To quantify the bundling process and explore how it depends on MT length, we measured the bundling time as the total observation time of MTs divided by the number of observed bundling events, $\left\langle t_{\mathrm{B}}\right\rangle=t_{\exp } / n$. We separated the measurements into groups with MT length $(\mathrm{R})$ greater than or less than $1 \mu \mathrm{m}$ and observed that the bundling was slower for longer MTs (for $R<1 \mu \mathrm{m},\left\langle t_{\mathrm{B}}\right\rangle=122 \pm 16 \mathrm{~min}$; for $R>1 \mu \mathrm{m},\left\langle t_{\mathrm{B}}\right\rangle=268 \pm 45 \mathrm{~min} ; N=72$ ). Slower bundling of longer MTs could be the result of smaller angular diffusion coefficients (for $R<1 \mu \mathrm{m}, D=(46 \pm 7) \times 10^{-4} \mathrm{rad}^{2} \mathrm{~s}^{-1}$; for $R>1 \mu \mathrm{m}, D=(9 \pm 1) \times 10^{-4} \mathrm{rad}^{2} \mathrm{~s}^{-1}$; see Appendix B and Supplemental Material Fig. 1 [38]), which makes the time it takes for MTs to get into close proximity to each other longer.

To test to what extent the MT cross-linker Ase1 affects the bundling process, we performed the measurements on mutant cells in which Ase1 was knocked out (denoted ase $1 \Delta$ ). Ase1/PRC1/MAP65 are passive MT cross-linkers known for bundling of antiparallel MTs in vivo and in vitro [12,13,36,40-43]. In fission yeast, Ase1 also plays a role in bundling parallel MTs. This was shown in meiotic prophase, where MTs originate from a single point with the same orientation [34,35], and bundling of these parallel MTs is reduced in the absence of Ase1 [36].

Ase1 also exhibits more complex behaviors, such as recruitment of Kinesin 6 during anaphase B, which might contribute to MT bundling as well [44]. In our experiments on ase $1 \Delta$ cells we observed occasional MT bundling events (see Supplemental Materials Figs. S2(a) and S2(b), Movie 4 , and Table I [38]), but the bundling time was significantly slower (for $R<1 \mu \mathrm{m},\left\langle t_{\mathrm{B}}\right\rangle=218 \pm 54 \mathrm{~min}$; for $R>1 \mu \mathrm{m}$, $\left.\left\langle t_{\mathrm{B}}\right\rangle=594 \pm 198 \mathrm{~min} ; N=43\right)$. Just like in the wild type cells, the bundling was slower for longer MTs. Thus, the efficiency of parallel bundle formation depends on several parameters, including MT length and the presence of MTs cross-linkers such as Ase1.

\section{B. Theory}

To explore the physical principles underlying the formation and stability of MT bundles, we introduce the pivot-andbond model [Fig. 2(a)]. In our model a MT pivots around a fixed point and eventually approaches the other MT, which is followed by accumulation of proteins bound to both MTs. These cross-linking proteins tie the MTs into a firm bundle.

In our model, we describe two MTs as thin rigid rods of fixed length with one end freely joint at the spindle pole, based on experimental observations [26,28]. The orientation of the first MT at time $t$ is described by a unit vector $\hat{\mathbf{r}}(t)$ [Fig. 2(b)]. The orientation of the unit vector changes as

$$
\frac{\mathrm{d} \hat{\mathbf{r}}}{\mathrm{d} t}=\omega \times \hat{\mathbf{r}},
$$

ensuring that the magnitude of $\hat{\mathbf{r}}$ does not change. The vector $\boldsymbol{\omega}$ denotes angular velocity of the MT. The other MT has a fixed orientation along the $\mathrm{z}$-axis in the direction of unit vector $\hat{\mathbf{z}}$. In the overdamped limit, the angular friction is balanced by the torque, $\mathbf{T}$, experienced by the MT:

$$
\gamma \omega=\mathbf{T}
$$

Here, $\gamma$ denotes the angular drag coefficient. We calculate the total torque as $\mathbf{T}=\boldsymbol{\tau}+\sigma(\hat{\mathbf{r}} \times \boldsymbol{\eta}(t))$, where the first and the second term represent the deterministic and the stochastic components, respectively. If the noise is caused by thermal fluctuations, as in fission yeast [26], $\boldsymbol{\eta}=\left(\eta_{i}\right), i=1,2,3$ is a three-dimensional Gaussian white noise where $i$ th and $j$ th components for times $t$ and $t^{\prime}$ obey $\left\langle\eta_{i}(t), \eta_{j}\left(t^{\prime}\right)\right\rangle=$ $\delta\left(t-t^{\prime}\right) \delta_{i, j}$, with $\delta\left(t-t^{\prime}\right)$ being the Dirac $\delta$ function and $\delta_{i, j}$ is the Kronecker $\delta$ function. The magnitude of the noise is 
(a) Tubulin-GFP Sid4(SPB)-GFP
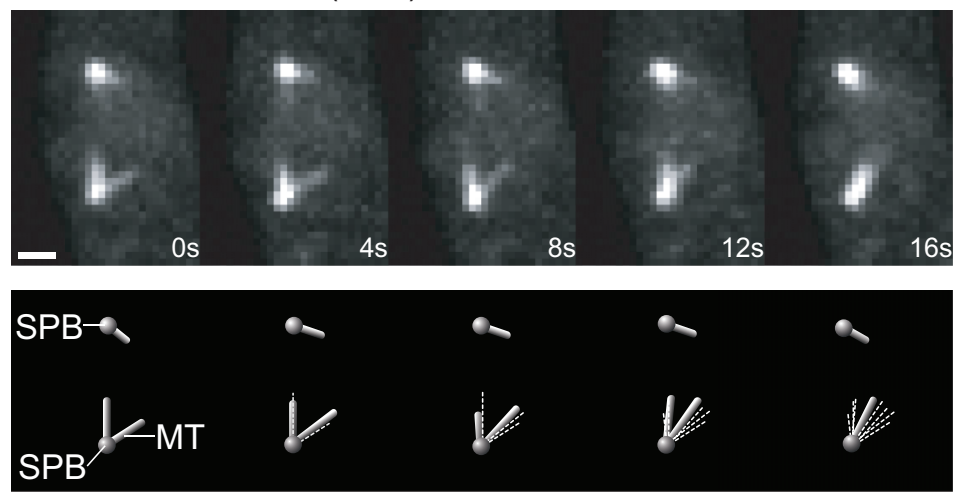

(b) Tubulin-GFP Sid4(SPB)-GFP
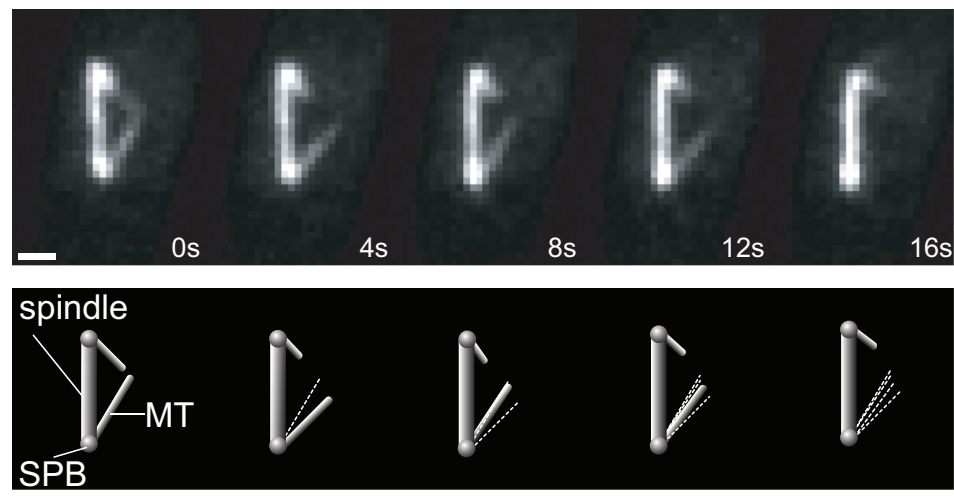

(e) Mal3-GFP Sid4(SPB)-GFP
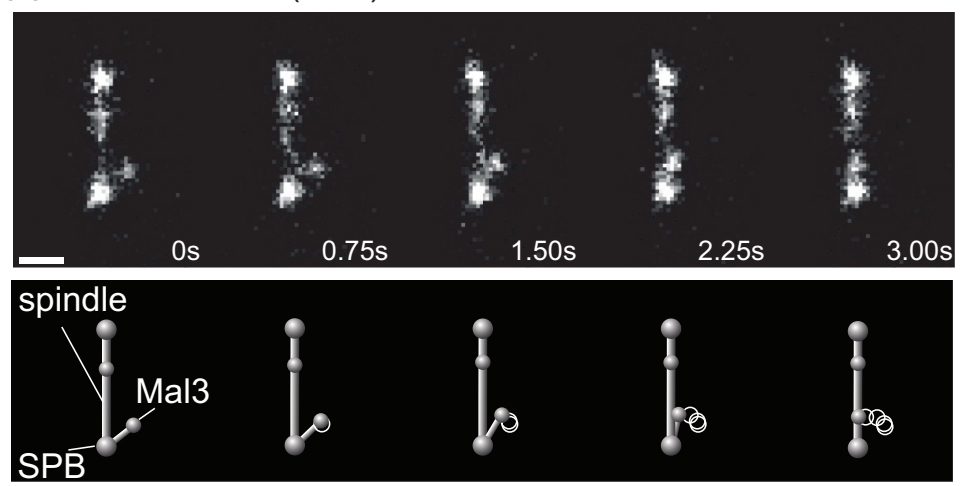

(b)

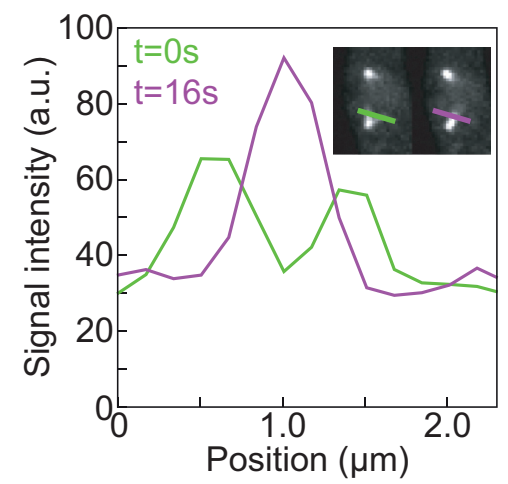

(d)

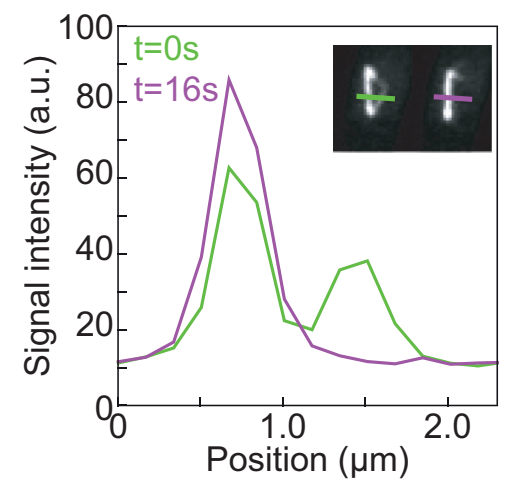

(f)

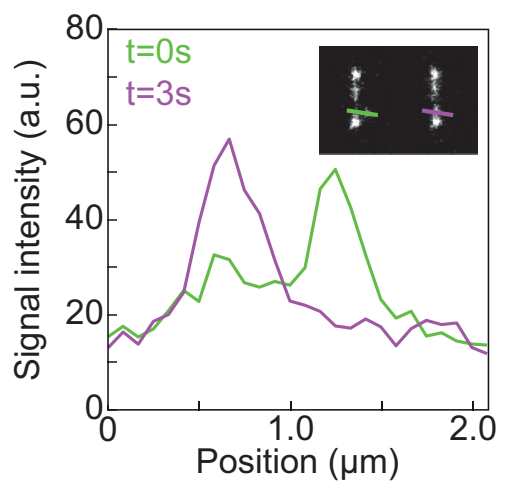

FIG. 1. Formation of MT bundles in S. pombe cells. (a) Time-lapse images and the corresponding drawings showing the formation of a parallel MT bundle at the lower spindle pole body in an $S$. pombe cell expressing tubulin-GFP and Sid4-GFP. (b) Measurement of the tubulin-GFP signal intensity of MTs before bundling (green curve in the graph, measured along the green line in the inset) and after bundling (magenta curve in the graph, measured along the magenta line in the inset). The measurements were done on the first and the last image in panel (a), respectively. After the contact between two MTs, the intensity of the tubulin-GFP signal increases. (c) Time-lapse images and the corresponding drawings showing a MT joining the bundle of spindle MTs in an S. pombe cell expressing tubulin-GFP and Sid4-GFP. (d) Measurements of the tubulin-GFP signal intensity of the spindle and MT before bundling (green curve in the graph, measured along the green line in the inset) and after bundling (magenta curve in the graph, measured along the magenta line in the inset). The measurements were done on the first and the last image in panel (c), respectively. After the bundling event, the intensity of the tubulin-GFP signal of the spindle increases. (e) Time-lapse images and the corresponding drawings showing a MT joining the bundle of spindle MTs in an S. pombe cell expressing Mal3-GFP and Sid4-GFP. (f) Measurements of the Mal3-GFP signal intensity of the spindle and MT before bundling (green curve in the graph, measured along the green line in the inset) and after bundling (magenta curve in the graph, measured along the magenta line in the inset). The measurements were done on the first and the last image in panel (e), respectively. After the bundling event, the intensity of the tubulin-GFP signal of the spindle increases. Scale bars in (a), (c), and (e) are $1 \mu \mathrm{m}$. In the drawings, microtubule orientations from the previous images are marked with white dashed lines. 
TABLE I. Values of the constant parameters used in this paper.

\begin{tabular}{llcc}
\hline \hline \multicolumn{1}{c}{ Parameter } & Value & Source \\
\hline$k$ & Spring stiffness & $100 \mathrm{pN} / \mu \mathrm{m}$ & Value for Eg5 [14] \\
$k_{\mathrm{a}}$ & Attachment rate & $0.01 \mathrm{~s}^{-1}$ & Value for Ase1 [13] \\
$k_{\mathrm{d} 0}$ & Detachment rate & $0.1 \mathrm{~s}^{-1}$ & Value for Ase1 [13] \\
$f_{0}$ & Critical force & $3 \mathrm{pN}$ & Value for kinesin-1 [58] \\
$y_{0}$ & Passive crosslinker rest length & $40 \mathrm{~nm}$ & Value for PRC1 [42,53] \\
$y_{0, \mathrm{~m}}$ & Motor rest length & $60 \mathrm{~nm}$ & Value for Kinesin-5 [54] \\
$D_{0}$ & Passive crosslinker diffusion constant & $0.05 \mu \mathrm{m}^{2} \mathrm{~s}^{-1}$ & Value for Ase1 [13,41] \\
$v_{0}$ & Motor velocity & $\pm 0.01 \mu \mathrm{m} / \mathrm{s}$ & Value for Cut7 [59] \\
$f_{0}$ & Motor stall force & $\pm 1.5 \mathrm{pN}$ & Value for cin8 [60] \\
$D$ & MT diffusion constant & $0.003 R^{-3} \mathrm{rad}^{2} \mathrm{~s}^{-1}$ & Fitted from Ref. [26] \\
\hline \hline
\end{tabular}

related to the angular drag coefficient, following the equipartition theorem, as $\sigma=\sqrt{2 k_{\mathrm{B}} T \gamma}$, with $k_{\mathrm{B}} T$ being the Boltzmann constant multiplied by the temperature. We introduce the angular diffusion coefficient, $D=k_{\mathrm{B}} T / \gamma$, and Eq. now reads

$$
\omega=\frac{D}{\mathrm{k}_{\mathrm{B}} T} \tau+\sqrt{2 D} \hat{\mathbf{r}} \times \eta(t) .
$$

In our model, the torque $\boldsymbol{\tau}$ in Eq. (3) is the consequence of forces exerted by cross-linking proteins connecting both MTs. If we denote the positions along the MTs as $\mathbf{z}=z \hat{\mathbf{z}}$ and $\mathbf{r}=$ $r \hat{\mathbf{r}}$, respectively, the torque contribution from cross-linking proteins is

$$
d \boldsymbol{\tau}(\mathbf{r}, \mathbf{z}, t)=\mathbf{r} \times \mathbf{f} d N(r, z, t),
$$

with $d N$ being the number of cross-linking proteins connecting the MT elements $[\mathbf{z}, \mathbf{z}+d \mathbf{z}]$ and $[\mathbf{r}, \mathbf{r}+d \mathbf{r}]$, while $\mathbf{f}$ is the force exerted by a single cross-linking protein. The elastic force is calculated as $\mathbf{f}=-k\left(\mathbf{y}-y_{0} \hat{\mathbf{y}}\right)$. Here $k$ is the Hookean spring constant, $\mathbf{y}=\mathbf{r}-\mathbf{z}$ is the elongation of the protein linking positions $\mathbf{r}$ and $\mathbf{z}$, with magnitude $y$ and direction $\hat{\mathbf{y}}=\mathbf{y} / y$, and $y_{0}$ is the relaxation length of the cross-linking protein. We describe the distribution of cross-linking proteins along the MTs by introducing the density, $\rho$, which obeys $d N(r, z, t)=\rho(r, z, t) d r d z$. To calculate the total torque we summed up all the attached cross-linking proteins:

$$
\boldsymbol{\tau}=k \int_{0}^{R} d r \int_{0}^{\infty} d z \rho \mathbf{r} \times \mathbf{z}\left(1-\frac{y_{0}}{|\mathbf{z}-\mathbf{r}|}\right),
$$

where we used $\mathbf{r} \times(\mathbf{z}-\mathbf{r})=\mathbf{r} \times \mathbf{z}$ and allowed the fixed MT to span the entire positive $z$ axis. When the total number of cross-linking proteins is large we can use the mean field limit and consider them continually distributed along the MT. In this limit, the cross-linking protein density is given by

$$
\frac{\partial \rho}{\partial t}=-\frac{\partial j_{\mathrm{r}}}{\partial r}-\frac{\partial j_{\mathrm{z}}}{\partial z}+k_{\mathrm{a}} c_{0} p(y)-k_{d}(r, z) \rho .
$$

Here, the currents describe the redistribution of cross-linking proteins along the MTs, $j_{r, z}=v_{r, z} \rho-D_{\mathrm{m}} \partial_{r, z} \rho$, where the two terms correspond to the drift and the diffusion of crosslinking proteins, respectively, with $v_{\mathrm{r}, z}$ being the drift velocity of cross-linking proteins along the respective MTs and $D_{m}$ being the variance of those velocities. In our model, the drift velocity depends only on component of the force parallel to the respective MT, $f_{\mathrm{r}}=\mathbf{f} \cdot \hat{\mathbf{r}}$ or $f_{\mathrm{z}}=\mathbf{f} \cdot \hat{\mathbf{z}}$, where the functional form differs for motor proteins and passive crosslinkers.

For passive crosslinkers, the velocities are calculated from the balance of the projections of the force and the friction of cross-linking proteins moving along the respective MT,

$$
\gamma_{0} v_{\mathrm{r}, \mathrm{z}}= \pm f_{\mathrm{r}, \mathrm{z}}
$$

where the coefficient of friction, $\gamma_{0}$, is related to the variance of the velocities, $D_{m}$, by the fluctuation-dissipation theorem, $\gamma_{0}=D_{0} / k_{\mathrm{B}} T$. In the case of active motors, we use a linear force-velocity relationship to obtain

$$
v_{\mathrm{r}, \mathrm{z}}=v_{0}\left[1 \pm \frac{f_{\mathrm{r}, \mathrm{z}}}{f_{0}}\right] \text {. }
$$

Here, $v_{0}$, and $f_{0}$ denote the velocity of an attached end of a motor at zero load and the stall force, respectively. The sign of the velocity at zero load and the stall force depend on the direction of movement for the motor (they are positive for plus-end directed motors and negative for minus-end directed motors).

The other two terms in Eq. (6) represent the cross-linking protein attachment and detachment. In our model, the attachment rate, $k_{a}$, is constant, but attachment is only possible if the elongation of the cross-linking protein is large enough to bridge the gap between the MTs. As long crosslinking proteins are in the nucleoplasm, their extension are in thermodynamic equilibrium with the Boltzmann distribution, $p=\sqrt{k /\left(2 \pi k_{B} T\right)} \exp \left[-k\left(y-y_{0}\right)^{2} / 2 k_{\mathrm{B}} T\right]$. Finally, we assume that the linear concentration of crosslinkers, $c_{0}$, is constant because their number is significantly greater than the number of attached ones. The detachment rate depends on the force experienced by the cross-linking proteins [45], $k_{d}(r, z)=k_{d 0} \exp \left[k y(r, z) / f_{\mathrm{c}}\right]$, with $f_{\mathrm{c}}$ being the critical force required for rupturing the MT-protein bond.

\section{Angular movement of microtubules for cross-linking proteins in the adiabatic approximation}

Equations (1)-(8) provide a complete description of the angular movement for the MT in the presence of cross-linking proteins, where the Eq. (7) applies to the passive crosslinkers, while Eq. (8) applies to the motor proteins. Because of the symmetry of the system, the rotation around the z-axis does not contribute to MT bundling, so we are only interested in the behavior of the angle between the MTs. Therefore, we 
(a)

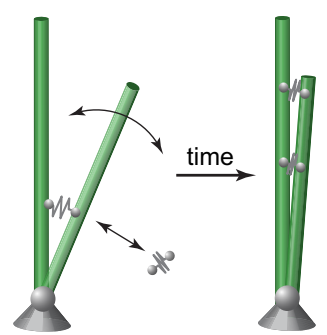

(c)

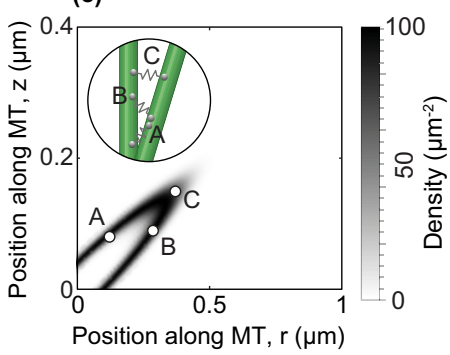

(e)

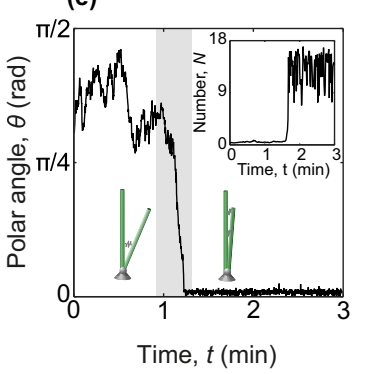

(b)

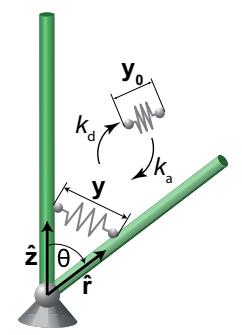

(d)
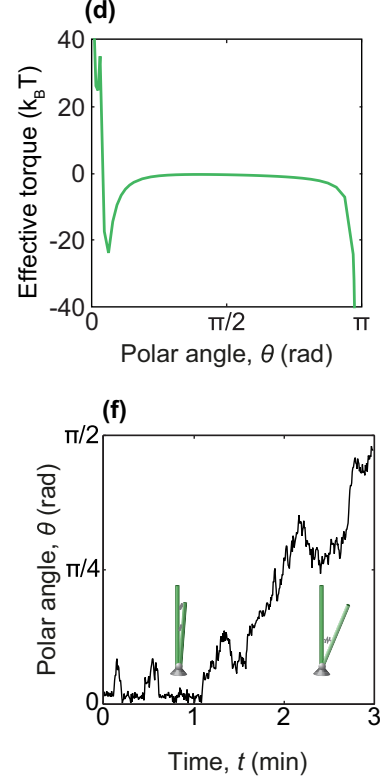

(g)

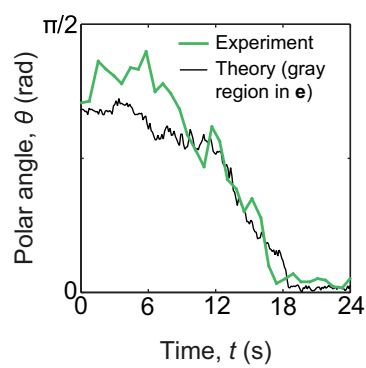

FIG. 2. Scheme of the model and numerical solutions. (a) Cartoon representation of the bundling process. Left, a MT (green rod) pivots around the spindle pole (gray ball). Right, when the MTs come into close proximity, cross-linking proteins (grey springs) attach and cause the MTs to from a bundle. (b) Scheme of the model. The orientations of two MTs are represented by the unit vectors $\hat{r}$ and z. Cross-linking proteins attach to and detach from MTs at rates $k_{a}$ and $k_{d}$, respectively. The elongation of the attached cross-linking protein is denoted $y$ and their relaxed length is $y_{0}$. The angle between the MTs is denoted $\theta$. (c) Density of passive crosslinkers. The inset shows the positions (drawn to scale) of crosslinkers that correspond to the points denoted on the main graph. Angle between the MTs is $\theta=0.3$ rad. (d) Effective torque caused by passive crosslinkers as a function of the angle between the MTs. (e) A sample path for the starting angle $\theta=1 \mathrm{rad}$, which shows a bundling event (around the 1 min mark). The crosslinker concentration is $c_{0}=60 \mu \mathrm{m}^{-1}$. (f) A sample path for the starting angle $\theta=0.01 \mathrm{rad, \text {whichshows }}$ an unbundling event (around the 1 min mark). (g) A sample of MT first parameterize the orientation of the MT given by the unit vector by $\hat{\mathbf{r}}(\theta, \phi)=(\sin \theta \cos \phi, \sin \theta \sin \phi, \cos \theta)$, where $\theta$ and $\phi$ denote the polar and azimuthal angle, respectively. In this parameterization, the equation of motion for the polar angle reads

$$
\partial_{t} \theta=\frac{D}{k_{\mathrm{B}} T} \tau_{\theta}+\sqrt{2 D} \eta(t),
$$

where $\boldsymbol{\tau}_{\theta}=(\hat{\mathbf{r}} \times \boldsymbol{\tau}) \cdot \hat{\boldsymbol{\theta}}+k_{\mathrm{B}} T \cot \theta$ is the effective torque in which the first term represents the projection of the torque exerted by the cross-linking proteins in the direction of the polar angle and the second term, $k_{\mathrm{B}} T \cot \theta$, is the spurious drift, which is the result of the coordinate transformation (derivation given in Appendix A).

To solve Eq. (5) and obtain the equation of motion for the angle between MTs, we need to calculate the density of the cross-linking proteins from Eq. (6). To reduce the complexity of our model, we use the adiabatic approximation, $\partial \rho / \partial t=0$. In this approximation, the number of attached cross-linking proteins, $N$, does not depend explicitly on time. For larger angles, $2 R \sin \frac{\theta}{2}>y_{0}$, the number of attached crosslinking proteins changes with the angle between the MTs, $N(\theta)=\frac{k_{\mathrm{a}} c_{0}}{k_{\mathrm{d} 0}} y_{0} \frac{\pi-\theta}{\sin \theta}$. For smaller angles the number is roughly constant, $N(0)=\frac{k_{\mathrm{a}} c_{0}}{k_{\mathrm{d} 0}} R$, but changes with the MT length. Both expressions are obtained by integrating the Eq. (6) over the MTs, which cancels out the cross-linking protein currents (see Appendix A).

\section{Distributions of passive crosslinkers and the resulting torques}

For passive crosslinkers, whose velocity along the MTs is given by Eq. (7), the density is given by

$$
\rho(r, z)=\frac{k_{\mathrm{a}} c_{0}}{k_{d}} \sqrt{k /\left(2 \pi k_{\mathrm{B}} T\right)} \exp \left[-\frac{k\left(y-y_{0}\right)^{2}}{2 k_{\mathrm{B}} T}\right] .
$$

The density of passive crosslinkers is shown in Fig. 2(c). Toward the spindle pole, their distribution is focused in the region in which the extension is close to $y_{0}$, as the crosslinkers prefer to arrange themselves so they are in a relaxed state (see points labeled A and B in Fig. 2(c)). Toward the MT tips, after the points at which the shortest distance is equal to the relaxed length of the crosslinkers, $y_{0}$, they are always extended (see point labeled $\mathrm{C}$ in Fig. 2(c)) and their distribution falls off rapidly.

Knowing the distributions of the cross-linking proteins (Eq. (10)), we can calculate how the torque exerted on the MTs depends on the angle between, by using the equation (5) to. The effective torque has negative values, which means it causes MT bundling, in a region where it dominates over the thermal motion of the MTs. This occurs at small angles where the MTs are close enough to each other for the crosslinkers to

angle time series obtained using light microscopy on cells with the Mal3-GFP label. Green line corresponds to the segment of the sample path in the shaded area in (e). Unless stated otherwise, calculations are done with $c_{0}=10 \mu \mathrm{m}^{-1}, R=1 \mu \mathrm{m}$ and other parameters shown in Table I. 
attach in large numbers far enough from the spindle pole to generate attractive torques that overcome the thermal motion of the MTs. For angles at which the distance between the MT tips is smaller than the length of the cross-linker, $2 R \sin \frac{\theta}{2}<$ $y_{0}$, all of the attached crosslinkers are compressed, creating positive effective torque that attempts to spread the MTs apart. For large angles, the effective torque is close to the value for free diffusion, $\tau_{\theta} \approx k_{\mathrm{B}} T \cot \theta$.

The sample paths for the polar angle in the presence of passive crosslinkers are obtained by numerically solving equation (9). We find that the MT performs random movement that spans a large space (Fig. 2(e)). However, the movement can become abruptly constrained in the vicinity of angle zero and these small angles correspond to a bundled state. The constrained movement near angle zero is a consequence of short range attractive forces exerted by the crosslinkers that accumulate in larger numbers when MTs are in close proximity (Fig. 2(e), inset). Our numerical solutions also show that, if the crosslinker concentration is sufficiently small, the constrained MT movement in the vicinity of angle zero can suddenly switch back to free random movement (Fig. 2(f)). Through numerical simulations, we find that thermally driven MT pivoting and elastic forces are sufficient to generate sample paths that qualitatively resemble formation of parallel bundles observed in the experiments (Fig. 2(g)).

\section{E. Bundle stability and bundling time in the presence of passive crosslinkers}

To explore the macroscopic properties of the bundling process, we will use the stationary distribution of the polar angles, $p_{\theta}=p_{0} e^{-\frac{U(\theta)}{k_{\mathrm{B}} T}}$, where $U(\theta)=-\int \tau_{\theta} \mathrm{d} \theta$ is the MTMT interaction potential and $p_{0}=1 / \int_{0}^{\pi} e^{-\frac{U(\theta)}{k_{\mathrm{B}} T}} \mathrm{~d} \theta$ is the normalization constant. For the case in which the interaction is mediated by passive crosslinkers, the potential can be calculated analytically (see Appendix A) and has the form $U(\theta)=-k_{\mathrm{B}} T\left(\theta_{\max }\left(\frac{\Theta\left(\theta-\theta_{\min }\right)}{\theta}+\frac{\Theta\left(\theta_{\min }-\theta\right)}{\theta_{\min }}\right)+\ln (\sin \theta)\right)$, where $\theta_{\min }=\frac{y_{0}}{R}$ is the local minimum representing the angle at which the distance between the MT tips is exactly equal the relaxed length of the crosslinker, and $\theta_{\max }=\frac{k_{a} c_{0}}{k_{\mathrm{d} 0}} y_{0} \pi$ is the local maximum of the potential, which is proportional to the conentrations of the crosslinkers. The potential has a well at small angles (Fig. 3(a)), but at large angles it the same as the potential for a freely diffusing MT, $U=-k_{\mathrm{B}} T \ln (\sin \theta)$ (Fig. 3(a), inset). The depth of the potential well in our model, and therefore the strength of the MT-MT interaction, depends on the attachment and detachment rates of crosslinkers, their concentration, as well as the relaxation length of the crosslinkers. The length of the MT determines the interaction range, but not the depth of the potential well. Note that the MT-MT interaction potential does not depend on the stiffness of the passive crosslinkers, even though the distribution does.

Next, we explored the stability of the bundles. Because MT bundles can be assembled and disassembled, as shown in Figs. 2(e) and 2(f), we treat the MT as a two state system. The position of the maximum of the normalized potential defines two regions, $\Theta_{\mathrm{B}} \equiv\left[0, \theta_{\max }\right]$ and $\Theta_{\mathrm{U}} \equiv\left[\theta_{\max }, \pi\right]$, which we term as the bundled and unbundled state, respectively. We define bundles as stable if the probability of finding the MT in (a)

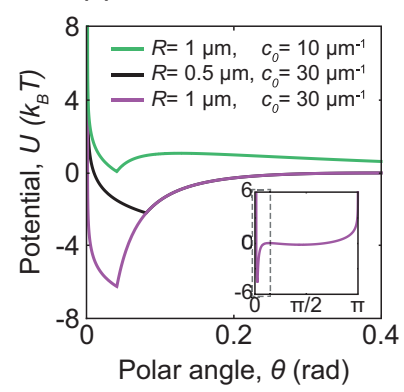

(c)

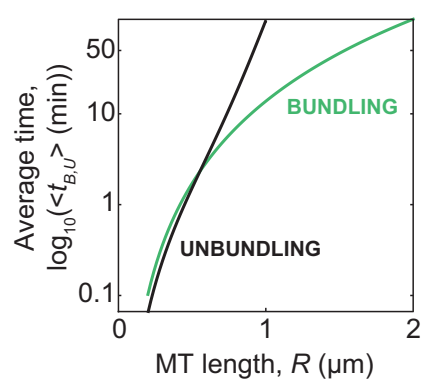

(b)

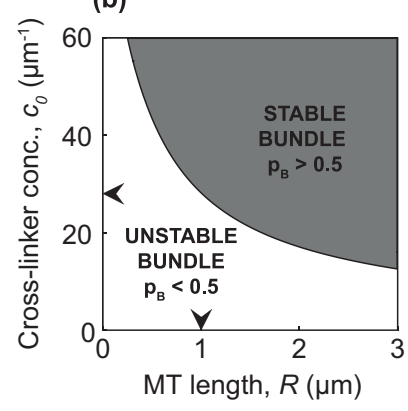

(d)

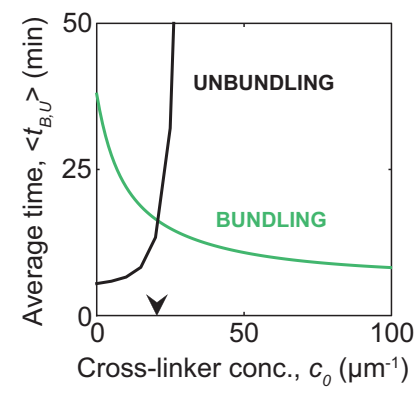

FIG. 3. Solutions of the model in the adiabatic approximation. (a) The effective potential as a function of the polar angle, for angles between $0 \mathrm{rad}$ and $0.4 \mathrm{rad}$. The inset shows the potential for all angles. Dashed square in the inset represents the region plotted in the large image. Values of the parameters used are given in the legend. (b) Stability diagram for plus-end directed motors. The gray area represents the region where bundles are stable, i.e., bundling is more probable than unbundling. (c) Logarithmic plot of the bundling (black) and unbundling (green) times as functions of MT length. The crosslinker concentration is $c_{0}=30 \mu \mathrm{m}^{-1}$. (d) Bundling (black) and unbundling (green) times as functions of crosslinker concentration. The MT length is $R=1 \mu \mathrm{m}$. Arrow represents the parameter values shown with arrows in (b). All calculations done with parameter values from Table I.

the bundled state, $P_{\mathrm{B}}$, is greater than the probability of finding it in the unbundled state, $P_{\mathrm{U}}$,

$$
P_{\mathrm{B}}>P_{\mathrm{U}} \text {. }
$$

The region in the parameter space where bundles are stable is shown in Fig. 3(c). We find that the bundles are unstable if MTs are too short or if there are not enough cross-linking proteins in the nucleoplasm.

Finally, we calculate how the MT bundling time depends on the parameters of the system. In the case of an isotropic distribution of initial MT orientations, the bundling time is calculated as $\left\langle t_{\mathrm{B}}\right\rangle=\frac{1}{2} \int_{\theta_{\min }}^{\pi}\langle t\rangle_{\theta, \theta_{\min }} \sin \theta \mathrm{d} \theta$, where $\langle t\rangle_{\theta, \theta_{\min }}$ is the first passage time from some initial angle $\theta$ to the angle $\theta_{\min }$ (ref. [46]). After solving these integrals numerically, we found that the bundling time, $\left\langle t_{\mathrm{B}}\right\rangle$, increases with MT length (green line in Fig. 3(c)). Note that the bundling time, $\left\langle t_{\mathrm{B}}\right\rangle$, is inversely proportional to $D$, which decreases with MT length [26], thus we expect that the bundling time increases with MT length. Additionally, the bundling time decreases as the cross-linker concentration increases (green line in Fig. 3(d)). We also calculate the unbundling time as $\left\langle t_{\mathrm{U}}\right\rangle=\frac{1}{2} \int_{\theta_{\min }}^{\pi} \mathrm{d} \theta \sin \theta\langle t\rangle_{\theta_{\min }, \theta}$, where $\langle t\rangle_{\theta_{\min }, \theta}$ is the first 
passage time from $\theta_{\min }$ to $\theta$. Unbundling time becomes longer than bundling time if the condition for bundle stability in equation (11) is fulfilled. Once this condition is satisfied, the bundling time greatly increases (black lines in Figs. 3(c) and 3(d)). For example, for a concentration that is only twice as large as the concentration for which stability is achieved, the bundling time increases 1000 times. Thus, it is unlikely that unbundling events would be observed in our biological system.

\section{F. Bundling in the presence of plus- and minus-end directed motors}

To explore the bundling process in the case when the interaction between MTs is mediated by plus-end directed motors, we change the force-velocity relationship for cross-linking proteins. For motor proteins, the force-velocity relationship is given by equation (8), which together with the equation (6) describes their distribution along the MTs. In the limit where the time scale of the motor movement is shorter than the time scale of motor detachment, $k v_{0} / f_{0} \gg k_{\text {off }}$, we write the density of the motors as the number of attached motors multiplied by their stationary probability distribution,

$$
\rho(r, z)=N(\theta) \frac{\exp \left[\frac{v_{0}}{D_{\mathrm{v}}}\left(r+z-\frac{k}{2 f_{0}}\left(y-y_{0}\right)^{2}\right)\right]}{\int_{0}^{R} \mathrm{~d} r \int_{0}^{\infty} \mathrm{d} z \exp \left[\frac{v_{0}}{D_{\mathrm{v}}}\left(r+z-\frac{k}{2 f_{0}}\left(y-y_{0}\right)^{2}\right)\right]}
$$

The plus-end directed motors walk toward the MT tips, so their distribution (Fig. 4(a)) is a Gaussian around the point of stall, which is the same on both MTs, $(r, z)=\left(u_{0}, u_{0}\right)$, with the stall point being $u_{0}=\left(y_{0}+\Delta y\right) / 2 \sin \frac{\theta}{2}$ [see point labeled A in Fig. 4(a)], and $\Delta y=f_{0} / k \sin \frac{\theta}{2}$. Moving away from this point toward either end of the MT [see point labeled B in Fig. 4(a)], or any tilting [see point labeled C in Fig. 4(a)] has lower probability of finding a motor in that configuration.

The plus-end directed motors generate strong bundling torque. Their torque is two orders of magnitude greater than the torque generated by the passive crosslinkers, for the same number of proteins [Fig. 4(b)]. This difference arises because the average motor is extended by $\Delta y$, whereas the average passive crosslinker is in a relaxed state. The potential has a similar shape to the one that occurs in the case of passive crosslinkers [Fig. 4(b), inset], but the well is much deeper and wider. The local maximum occurs only in the case of small motor concentrations, $c_{0}<1 \mu \mathrm{m}^{-1}$ for $R>0.3 \mu \mathrm{m}$ and other parameters taken from Table I, while it vanishes for higher concentrations, which corresponds to the case when the effective torque is attractive for all angles.

Next we explore the stability of the bundles caused by plusend directed motors, in the same way as we did for passive crosslinkers, by evaluating the condition given in Eq. (11), but with the motor distribution given in Eq. (12). In the presence of plus-end directed motors, bundle stability is achieved for most of the points in the parameter space, except for very short MTs and very low concentrations of motors [Fig. 4(e)]. For example, with the concentration being only $c_{0}=1 \mu \mathrm{m}^{-1}$, bundles are stable for all MTs with $R>0.1 \mu \mathrm{m}$.

Finally, the distribution of minus-end directed motors is obtained by using a negative velocity in Eq. (12). It has two

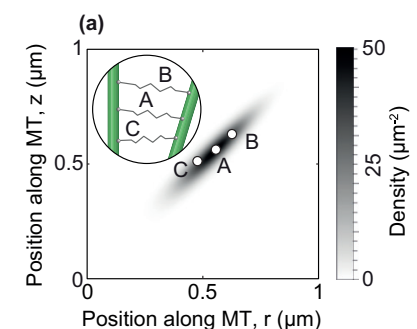

(b)

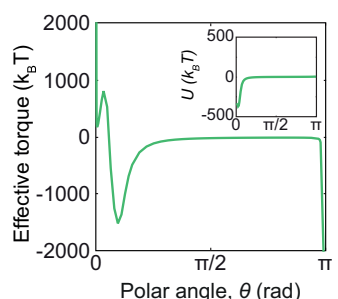

(c)

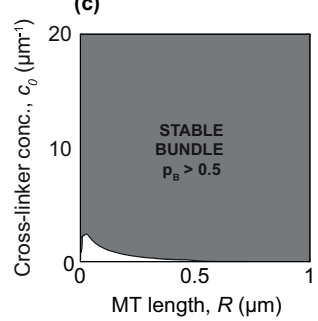

FIG. 4. Solutions of the model for motors. (a) Density of plusend directed motors. The inset shows the positions (drawn to scale) of motors that correspond to the points denoted on the main graph. Angle between the MTs is $\theta=0.3 \mathrm{rad}$. (b) Effective torque caused by plus-end directed motors as a function of the angle between the MTs. The inset shows the potential. (c) Stability diagram for plus-end directed motors. The gray area represents the region where bundles are stable, i.e., bundling is more probable than unbundling. (d) Density of minus-end directed motors. The inset shows the positions (drawn to scale) of motors that correspond to the points denoted on the main graph. Angle between the MTs is $\theta=0.3 \mathrm{rad}$. (e) Effective torque caused by minus-end directed motors as a function of the angle between the MTs. The inset shows the potential. All calculations are done with $c_{0}=10 \mu \mathrm{m}^{-1}, R=1 \mu \mathrm{m}$ and other parameters shown in Table I.

peaks near the spindle pole, at points $(r, z)=\left(y_{0}-\Delta y, 0\right)$ [Fig. 4(d), point labeled A] and $(r, z)=\left(0, y_{0}-\Delta y\right)$ (point labeled $\mathrm{B}$ ). This is because one of their ends moves all the way to the spindle pole, with the other one is being compressed by $\Delta y$ along the MT due to its walking. The minus-end directed motors produce negligible torques [Fig. 4(e)], because they mostly accumulate so they are parallel to the MTs. Thus, we do not expect any bundles to form in this case.

\section{DISCUSSION}

In conclusion, the pivot-and-bond model implies that passive processes, thermally driven motion of the MTs and passive crosslinkers, are sufficient to describe the formation of parallel MT bundles. As expected, the stability of formed bundles increases and the time it takes for them to form decreases as the concentration of passive crosslinkers increases. The bundling time is longer for longer MTs, as their angular 
diffusion become slower and it takes them longer to find their pair, but the bundles formed by longer MTs are more stable due to the larger total number of crosslinkers being attached. Thus, the model provides a plausible mechanism for MT bundling observed in our experiments.

Along with passive crosslinkers, we modeled active motors and their effects on MT bundling. Our theory predicts that the plus-end motors exert much larger attractive torques than passive crosslinkers because they accumulate closer to the MT tips and are always extended. Even for very short MTs and only a couple of plus-end motors attached, the MTs will form stable bundles. This result opens up interesting questions of whether, in addition to passive crosslinkers, the plus-end directed motors contribute to formation of parallel bundles.

Using the minimal model approach, we gain a deeper understanding of the mesoscopic properties of the bundling process, such as bundle stability and average bundling time, as well as predict their behavior for a wide range of parameter values. This approach is complementary to more exhaustive and detailed methods such as large-scale numerical simulations (for example, Refs. [25,47]).

Although the pivot-and-bond model applies to fission yeast, the approach used here could also be applied to other biological systems. In higher eukaryotes, where a large fraction of MTs is nucleated at the centrosome, short MTs growing from the centrosome may perform substantial angular motion and create parallel bundles upon contact. In addition, MTs can be nucleated at sites positioned along the pre-existing mother MT [48-50]. In meiotic Xenopus egg extracts, these new MTs grow at small angles and with the same polarity as the mother MT, which makes them suitable to generate parallel MT bundles such as k-fibers [51]. Quantification of the angular movement of these MTs will show to which extent it contributes to the formation of bundles.

The number of MT bundles depends on the cell type. The pivot-and-bond mechanism presented in this work is relevant for the formation of a single bundle and thus can be directly applied to simple rod-like spindles such as a yeast spindle. In higher eukaryotes, however, the mitotic spindle is comprised of multiple parallel and antiparallel MT bundles that remain separated during mitosis. Future work will uncover the mechanisms underlying the formation and maintenance of such complex MT-based structures.

\section{ACKNOWLEDGMENTS}

N.P. and I.M.T. designed and supervised the project. M.P. and N.P. developed the theory. L.W. carried out the experiments and A.M. analyzed the experimental data and performed some of the experiments. N.P., M.P., and I.M.T. wrote the manuscript with input from L.W. and A.M. We thank D. Radić and V. Despoja for comments on the manuscript, as well as I. Ban and M. Glunčić for valuable discussions and advice regarding our theoretical model; F. Elsner from the Electronics Service of MPI-CBG for building the thermoelectric device; J. Millar for yeast strains; Light Microscopy Facility of MPI-CBG for help with microscopy; J. Brugués, T. M. Franzmann, A. Garcia Ulloa, and the rest of the Tolić lab for discussion and advice; and I. Šarić for editing the figures. We acknowledge the Unity through Knowledge Fund (UKF),
German Research Foundation (DFG), European Research Council (ERC), and QuantiXLie Centre of Excellence, a project cofinanced by the Croatian Government and European Union through the European Regional Development Fund the Competitiveness and Cohesion Operational Programme (Grant No. KK.01.1.1.01.0004). The data that support the findings of this study are available from the corresponding authors upon reasonable request. Contact N.P. for information on the theory presented and I.M.T. with requests related to the experimental measurements.

\section{APPENDIX A: THEORY}

\section{Equation of motion in spherical coordinates}

Equation (1), for the angular velocity given by Eq. (3), reads

$$
\frac{d \hat{\mathbf{r}}}{d t}=\left(\frac{D}{k_{\mathrm{B}} T} \boldsymbol{\tau}+\sqrt{2 D} \hat{\mathbf{r}} \times \eta(t)\right) \times \hat{\mathbf{r}} .
$$

Here $\boldsymbol{\eta}=\left(\eta_{i}\right)$ is a three-dimensional Gaussian white noise in some Euclidian coordinates $i, j=x, y, z$, where $i$ th and $j$ th components for times $t$ and $t^{\prime}$ obey $\left\langle\eta_{i}(t), \eta_{j}\left(t^{\prime}\right)\right\rangle=$ $\delta\left(t-t^{\prime}\right) \delta_{i, j}$. In Cartesian coordinates, Eq. (A1) can be written componentwise as

$$
\begin{aligned}
{\left[\begin{array}{c}
\hat{\hat{r}}_{\mathrm{x}} \\
\dot{\hat{r}}_{\mathrm{y}} \\
\dot{\hat{r}}_{\mathrm{z}}
\end{array}\right]=} & \frac{D}{k_{\mathrm{B}} T}\left[\begin{array}{ccc}
0 & -\hat{r}_{\mathrm{z}} & \hat{r}_{\mathrm{y}} \\
\hat{r}_{\mathrm{z}} & 0 & -\hat{r}_{\mathrm{x}} \\
-\hat{r}_{\mathrm{y}} & \hat{r}_{\mathrm{x}} & 0
\end{array}\right]^{\mathbf{T}}\left[\begin{array}{c}
\tau_{\mathrm{x}} \\
\tau_{\mathrm{y}} \\
\tau_{\mathrm{z}}
\end{array}\right] \\
& +\sqrt{2 D}\left[\begin{array}{ccc}
0 & -\hat{r}_{\mathrm{z}} & \hat{r}_{\mathrm{y}} \\
\hat{r}_{\mathrm{z}} & 0 & -\hat{r}_{\mathrm{x}} \\
-\hat{r}_{\mathrm{y}} & \hat{r}_{\mathrm{x}} & 0
\end{array}\right]\left[\begin{array}{ccc}
0 & -\hat{r}_{\mathrm{z}} & \hat{r}_{\mathrm{y}} \\
\hat{r}_{\mathrm{z}} & 0 & -\hat{r}_{\mathrm{x}} \\
-\hat{r}_{\mathrm{y}} & \hat{r}_{\mathrm{x}} & 0
\end{array}\right]^{\mathbf{T}} \\
& \times\left[\begin{array}{c}
\eta_{\mathrm{x}} \\
\eta_{\mathrm{y}} \\
\eta_{\mathrm{z}}
\end{array}\right]
\end{aligned}
$$

where a cross-product is expressed as the product of a skewsymmetric matrix (shorter notations $\left[\hat{\mathbf{r}}_{\times}\right]_{\mathrm{C}}$ and $\left[\hat{\mathbf{r}}_{\times}\right]_{\mathrm{C}}^{\mathbf{T}}$, index $C$ denotes the Cartesian coordinates) and a vector. The dot represents derivation over time. Since we are interested in the angle between microtubules, we parameterize the orientation of the MT by using the polar angle $\theta$ and the azimuthal angle, $\hat{\mathbf{r}}_{\mathrm{C}}(\theta, \phi)=(\sin \theta \cos \phi, \sin \theta \sin \phi, \cos \theta)$. The Cartesian components of the unit vector and its differentials can be written in terms of angles as

$$
\begin{aligned}
{\left[\begin{array}{c}
\hat{r}_{\mathrm{x}} \\
\hat{r}_{\mathrm{y}} \\
\hat{r}_{\mathrm{z}}
\end{array}\right]=} & {\left[\begin{array}{c}
\sin \theta \cos \phi \\
\sin \theta \sin \phi \\
\cos \theta
\end{array}\right],\left[\begin{array}{c}
\dot{\hat{r}}_{\mathrm{x}} \\
\dot{\hat{r}}_{\mathrm{y}} \\
\dot{\hat{r}}_{\mathrm{z}}
\end{array}\right] } \\
= & {\left[\begin{array}{c}
\cos \theta \cos \phi \dot{\theta}-\sin \theta \sin \phi \dot{\varphi} \\
\cos \theta \sin \phi \dot{\theta}+\sin \theta \cos \phi \dot{\varphi} \\
-\sin \theta \dot{\theta}
\end{array}\right] . }
\end{aligned}
$$

Next, we introduce spherical unit vectors, $\hat{\mathbf{e}}_{\mu}$, with indices $\mu=r, \theta, \phi$. Transformation of Cartesian to spherical coordinates is given by $\hat{\mathbf{e}}_{\mu}=\mathcal{R}_{\mu i} \hat{\mathbf{e}}_{i}$, where $\hat{\mathbf{e}}_{i}$ are Cartesian unit vectors. The transformation matrix reads

$$
\mathcal{R}=\left[\begin{array}{ccc}
\sin \theta \cos \phi & \sin \theta \sin \phi & \cos \theta \\
\cos \theta \cos \phi & \cos \theta \sin \phi & -\sin \theta \\
-\sin \phi & \cos \phi & 0
\end{array}\right] .
$$


Vectors and tensors transform as $\dot{\hat{\mathbf{r}}}_{S}=\mathcal{R} \dot{\hat{\mathbf{r}}}_{C}$ and $\left[\hat{\mathbf{r}}_{\times}\right]_{S}=$ $\mathcal{R}\left[\hat{\mathbf{r}}_{\times}\right]_{C} \mathcal{R}^{\mathbf{T}}$, respectively, where index $S$ denotes spherical coordinates. Applying the transformation to all the terms in Eq. (A2), we write

$$
\dot{\hat{\mathbf{r}}}_{S}=\left[\hat{\mathbf{r}}_{\times}\right]_{S}^{\mathbf{T}} \boldsymbol{\tau}_{S}+\sqrt{2 D}\left[\hat{\mathbf{r}}_{\times}\right]_{S}\left[\hat{\mathbf{r}}_{\times}\right]_{S}^{\mathbf{T}} \boldsymbol{\eta}_{S},
$$

where we used $\mathcal{R}^{\mathrm{T}}=\mathcal{R}^{-1}$. After using the parameterization in Eq. (A3) and doing simple algebra, Eq. (A5) can be written as

$$
\begin{aligned}
{\left[\begin{array}{c}
\dot{\theta} \\
\dot{\phi}
\end{array}\right]=} & \frac{D}{k_{\mathrm{B}} T}\left[\begin{array}{l}
\tau \\
0
\end{array}\right] \\
& +\sqrt{2 D}\left[\begin{array}{ccc}
\cos \theta \cos \phi & \cos \theta \sin \phi & -\sin \theta \\
-\frac{\sin \phi}{\sin \theta} & \frac{\cos \phi}{\sin \theta} & 0
\end{array}\right] \circ\left[\begin{array}{c}
\eta_{\mathrm{x}} \\
\eta_{\mathrm{y}} \\
\eta_{\mathrm{z}}
\end{array}\right] .
\end{aligned}
$$

Since we used standard algebra to obtain Eq. (A6), it should be interpreted in the Stratonovich sense (denoted by $\circ$ ). To obtain the equivalent Ito equation, we start by re-writing the noise term as $\sum_{i=x, y, z} \mathbf{V}_{i} d W_{i}$, with vectors $\mathbf{V}_{i}$ being

$$
\begin{aligned}
& \mathbf{V}_{x}=\sqrt{2 D}\left[\begin{array}{c}
\cos \theta \cos \phi \\
-\frac{\sin \phi}{\sin \theta}
\end{array}\right], \quad \mathbf{V}_{y}=\sqrt{2 D}\left[\begin{array}{c}
\cos \theta \sin \phi \\
\frac{\cos \phi}{\sin \theta}
\end{array}\right], \\
& \mathbf{V}_{z}=\sqrt{2 D}\left[\begin{array}{c}
-\sin \theta \\
0
\end{array}\right] .
\end{aligned}
$$

We then use the Itō-Stratonovich transformation formula to calculate the spurious drift term in the Ito interpretation as $(1 / 2) \sum_{i=x, y, z} \nabla_{\mathbf{V}_{i}} \mathbf{V}_{i}$, with $\nabla_{\mathbf{V}_{i}} \mathbf{V}_{i}$ being the directional derivative of $\mathbf{V}_{i}$ along itself [52]. We use the independence of the components of the noise vector along different Cartesian axes $\eta_{i}$ to write their linear combinations that correspond to the noise vector components along the radial coordinates:

$$
\begin{aligned}
& \eta_{\theta}=\cos \theta \cos \varphi \eta_{\mathrm{x}}+\cos \theta \sin \varphi \eta_{\mathrm{y}}-\sin \theta \eta_{\mathrm{z}}, \\
& \eta_{\phi}=-\sin \varphi \eta_{\mathrm{x}}+\cos \varphi \eta_{\mathrm{y}} .
\end{aligned}
$$

It is easy to show that $\eta_{\theta}$ and $\eta_{\varphi}$ have the properties $\left\langle\eta_{i}\right\rangle=0$ and $\left\langle\eta_{i}, \eta_{j}\right\rangle=\delta_{i, j}$ for $i, j \in\{\theta, \varphi\}$, i.e., they are Gaussian white noises with unit variance. Finally, we obtain the equation of motion for the unit vector in spherical coordinates:

$$
\left[\begin{array}{c}
\dot{\theta} \\
\dot{\phi}
\end{array}\right]=D\left[\begin{array}{c}
\tau / k_{\mathrm{B}} T \\
0
\end{array}\right]+D\left[\begin{array}{c}
\cot \theta \\
0
\end{array}\right]+\sqrt{2 D}\left[\begin{array}{c}
\eta_{\theta} \\
\eta_{\phi} / \sin \theta
\end{array}\right] .
$$

The first term on the right-hand side is the contribution from crosslinkers, the second one is the spurious drift term calculated using directional derivatives of vectors in Eq. (A7), and the last one is the noise term for angular variables. The first component in Eq. (A9) is the equation of motion for the polar angle $\theta$ given in Eq. (7).

\section{Density of cross-linking proteins}

Equation (6) describes the attachment and detachment of crosslinkers, as well as their movement along the MTs. This equation does not have an analytical solution because the density depends on the polar angle, which is stochastic in nature. To solve this problem, we use the adiabatic approximation, $\partial \rho / \partial t=0$, which holds if the change in cross-linker distribution is faster than the movement of MTs. In this approximation Eq. (6) becomes

$$
\begin{aligned}
& -\frac{\partial j_{r}(r, z)}{\partial r}-\frac{\partial j_{z}(r, z)}{\partial z} \\
& +k_{a} c_{0} \sqrt{\frac{k}{2 \pi k_{\mathrm{B}} T}} \exp \left[\frac{-k\left(y-y_{0}\right)^{2}}{2 k_{\mathrm{B}} T}\right] \\
& -k_{\mathrm{d} 0} \exp \left[\frac{k\left(y-y_{0}\right)}{f_{\mathrm{c}}}\right] \rho=0,
\end{aligned}
$$

where $y(r, z)=\sqrt{r^{2}+z^{2}-2 r z \cos \theta}$ is the value of the cross-linker elongation. The currents written explicitly are

$$
\begin{aligned}
j_{r} & =-D_{m}\left[\frac{k}{k_{\mathrm{B}} T}(r-z \cos \theta)\left(1-\frac{y_{0}}{y}\right)+\frac{\partial}{\partial r}\right] \rho \\
j_{z} & =-D_{m}\left[\frac{k}{k_{\mathrm{B}} T}(z-r \cos \theta)\left(1-\frac{y_{0}}{y}\right)+\frac{\partial}{\partial z}\right] \rho
\end{aligned}
$$

In the $r$ and $z$ coordinate system, the currents $j_{r}$ and $j_{z}$ depend on both coordinates, so it is useful to transform the coordinates into a coordinate system in which coupling in the term $z-r \cos \theta$ disappears. Thus, we introduce coordinates $u=$ $(r+z) / 2$ and $w=(r-z) / 2$. These coordinates also have a physical interpretation, the first one describes the position of the cross-linker's center of mass and the second one describes the "tilt" of the cross-linker. In these coordinates, Eq. (A10) becomes

$$
\begin{aligned}
& -\left(\frac{\partial j_{u}}{\partial u}+\frac{\partial j_{w}}{\partial w}\right)+k_{\mathrm{a}} c_{0} \sqrt{\frac{k}{2 \pi k_{\mathrm{B}} T}} \exp \left[\frac{-k y_{0}^{2}\left(\frac{y}{y_{0}}-1\right)^{2}}{2 k_{\mathrm{B}} T}\right] \\
& -k_{d 0} \exp \left[\frac{k\left(y-y_{0}\right)}{f_{\mathrm{c}}}\right] \rho(u, w)=0
\end{aligned}
$$

where $y(u, w)=\sqrt{4 \sin ^{2} \frac{\theta}{2} u^{2}+4 \cos ^{2} \frac{\theta}{2} w^{2}}$ is the value of the cross-linker elongation in the new coordinates. The term $\sqrt{k /\left(2 \pi k_{\mathrm{B}} T\right)}$ comes from integrating the Boltzmann factor over all possible cross-linker elongations. The currents are now

$$
\begin{aligned}
& j_{u}=\frac{D_{m}}{2}\left[\frac{4 k}{k_{B} T} \sin ^{2} \frac{\theta}{2} u\left(1-\frac{y_{0}}{y}\right)-\frac{\partial}{\partial u}\right] \rho, \\
& j_{w}=\frac{D_{m}}{2}\left[\frac{4 k}{k_{B} T} \cos ^{2} \frac{\theta}{2} w\left(1-\frac{y_{0}}{y}\right)-\frac{\partial}{\partial w}\right] \rho
\end{aligned}
$$

Equations (A12) and (A13) provide an implicit definition of cross-linker density required for calculating the effective torque in Eq. (5), which in the $u$ and $w$ coordinates reads

$$
\begin{aligned}
\tau_{\theta}= & -2 k \sin \theta\left[\int_{0}^{\frac{R}{2}} d u \int_{-u}^{u} d w \rho(u, w)\left(u^{2}-w^{2}\right)\left(1-\frac{y_{0}}{y}\right)\right. \\
& \left.+\int_{\frac{R}{2}}^{\infty} d u \int_{u-R}^{u} d w \rho(u, w)\left(u^{2}-w^{2}\right)\left(1-\frac{y_{0}}{y}\right)\right] \\
& +k_{\mathrm{B}} T \cot \theta .
\end{aligned}
$$

Hence, our goal is to calculate the torque in Eq. (A14) by finding a suitable approximation for the cross-linker density. In the limit of a large MT, the second term in Eq. (A14) can be neglected. Next, to simplify our calculations, we consider 
the homogeneous part of Eq. (A12) only. We also employ symmetries of our system. By applying the finite integral $\int_{-u}^{u} d w$ we integrate the homogeneous part of Eq. (A12). The density $\rho(u, w)$ is an even function in $w$ because the system is invariant to switching the MTs. Thus, $j_{w}$ is an odd function in $w$ and $\int_{-u}^{u} j_{w} \mathrm{~d} w$ will vanish. We expand the term $\left(1-y_{0} / y\right)$ around the point $(u, w)=\left(y_{0} /\left(2 \sin \frac{\theta}{2}\right), 0\right)$. Because we are interested in finding the dominant contribution, we calculate the first order terms only. The integral reads

$$
\begin{gathered}
-D_{m} \frac{\partial}{\partial u}\left[\frac{k}{4 k_{\mathrm{B}} T} \sin ^{2} \frac{\theta}{2}\left(u-\frac{y_{0}}{2 \sin \frac{\theta}{2}}\right)-\frac{\partial}{\partial u}\right] \int_{-u}^{u} \rho d w \\
-\int_{-u}^{u} k_{d}(u, w) \rho d w=0 .
\end{gathered}
$$

To be consistent, we also expand the detachment term into a series up to the first order: $k_{d}(u, w)=k_{d 0}+k_{\mathrm{u}} u+k_{w} w+$ $\mathcal{O}_{2}(u, w)$. We now introduce a function $Q(u)=\int_{-u}^{u} \rho d w$ and obtain an ordinary differential equation for $Q(u)$ :

$$
\begin{aligned}
& -D_{m} \frac{\partial}{\partial u}\left[\frac{k}{4 k_{B} T} \sin ^{2} \frac{\theta}{2}\left(-u+\frac{2 u^{2} \sin \frac{\theta}{2}}{y_{0}}\right) Q-\frac{\partial Q}{\partial u}\right] \\
& -k_{d 0} Q-k_{\mathrm{u}} u Q=0 .
\end{aligned}
$$

The term $k_{w} \int_{-u}^{u} w \rho d w$ vanished for the odd function $w \rho(u, w)$. We use the expansion $Q=Q_{1} u+Q_{2} u^{2}+Q_{3} u^{3}+$ $\mathcal{O}_{4}(u)$ and group the terms associated with $u^{0}$ and $u^{1}$. The recursion relations for the coefficients of the expansion are

$$
\begin{aligned}
Q_{2} & =0, \\
D_{m} \frac{k}{2 k_{\mathrm{B}} T} \sin ^{2} \frac{\theta}{2} Q_{1}+6 D_{m} Q_{3}-k_{d 0} Q_{1} & =0 .
\end{aligned}
$$

In Eq. (A17), contributions from diffusion and detachment appear in a direct competition because they are both multiplying the same recursion term. We obtain that the diffusion term is dominant in the case $\left(k D_{m} / k_{\mathrm{B}} T\right)(1-\cos \theta) \gg k_{d 0}$. This condition holds for all angles $\theta \gg 10^{-3}$ if we use the parameters values from the Table I. Therefore, we use the approximation $k_{d}(r, z)=k_{d 0}$. In that case Eq. (A13) has an analytical solution:

$$
\rho(u, w)=\frac{k_{a} c_{0}}{k_{d 0}} \sqrt{\frac{k}{2 \pi k_{\mathrm{B}} T}} \exp \left[-\frac{k}{2 k_{\mathrm{B}} T}\left(y(u, w)-y_{0}\right)^{2}\right] .
$$

To solve the inner integrals in Eq. (A14), we make another coordinate transformation $(u, w) \rightarrow(y, \alpha)$, where $y$ is the elongation defined as before of the crosslinker and $\alpha$ is an angular variable, so that $u=y \cos \alpha / 2 \sin \frac{\theta}{2}$ and $w=$ $y \sin \alpha / 2 \cos \frac{\theta}{2}$. The integration region in Eq. (A14) can be approximated as a circular sector of radius $R / 2 \sin \frac{\theta}{2}$ and angle in range $\pm \tan ^{-1}\left(\cot \frac{\theta}{2}\right)= \pm(\pi-\theta) / 2$. This approximation holds unless the angle is small enough that the distance between the MT tips is less than the length of the crosslinker, $y_{0}<R / 2 \sin \frac{\theta}{2}$, and in this case we assume that no crosslinkers attach and no torque is exerted. For convenience, we will use the fact that the torque is significant only for small angles $\theta \ll 1$, so $R / 2 \sin \frac{\theta}{2} \rightarrow \infty$. Equation (A14) now reads

$$
\begin{aligned}
\tau_{\theta}= & -\frac{k}{2} \Theta\left(\sin \frac{\theta}{2}-\frac{y_{0}}{2 R}\right) \int_{0}^{\infty} \exp \left[-\frac{k}{2 k_{\mathrm{B}} T}\left(y-y_{0}\right)^{2}\right] \\
& \times \mathrm{y}^{2}\left(y-y_{0}\right) d y \int_{-\frac{\pi-\theta}{2}}^{\frac{\pi-\theta}{2}}\left[\left(\frac{\cos \alpha}{\sin \frac{\theta}{2}}\right)^{2}-\left(\frac{\sin \alpha}{\cos \frac{\theta}{2}}\right)^{2}\right] d \alpha \\
& +k_{\mathrm{B}} T \cot \theta .
\end{aligned}
$$

Both integrals can be solved analytically, yielding the torque

$$
\begin{aligned}
\tau_{\theta}= & -\frac{k_{a} c_{0}}{k_{d 0}} y_{0} \frac{1-(\theta-\pi) \sin \theta}{\sin \theta} \Theta\left(\sin \frac{\theta}{2}-\frac{y_{0}}{2 R}\right) \\
& +k_{\mathrm{B}} T \cot \theta .
\end{aligned}
$$

Integrating the torque with respect to $\theta$ and taking the negative value yields the effective MT-MT interaction potential, which is then simplified using the small angle approximation,

$$
U(\theta)=-\frac{k_{a} c_{0}}{k_{d 0}} y_{0} \pi\left[\frac{\Theta\left(\theta-\frac{y_{0}}{R}\right)}{\theta}+\frac{\Theta\left(\frac{y_{0}}{R}-\theta\right)}{\frac{y_{0}}{R}}\right]-\ln (\sin \theta) .
$$

The local extremes of this potential are $\theta_{\max }=\frac{k_{a} c_{0}}{k_{d 0}} y_{0} \pi$ and $\theta_{\min }=\frac{y_{0}}{R}$, as discussed in the main text. The generalized potential can be used to map macroscopic values such as bundling probability and bundling time onto the parameter space.

In the case where the cross-linking proteins are plusend directed motors, whose velocity is given by Eq. (8), we can use the same coordinate transformation as for passive crosslinkers. Furthermore, the distribution is close to a Gaussian centered at the point $(u, w)=\left(u_{0}, 0\right)$, with $u_{0}=$ $\left(y_{0}+\frac{f_{0}}{k \sin \frac{\theta}{2}}\right) / 2 \sin \frac{\theta}{2}$ and very narrow along the $w$ coordinate [Fig. 4(a)]. This allows us to roughly approximate the density of the plus-end directed motors given in Eq. (12) as

$$
\rho(u, w)=N(\theta) \frac{\exp \left[-\frac{k v_{0}}{f_{0} D_{\mathrm{v}}} \sin \frac{\theta}{2}\left(u-u_{0}\right)^{2}\right] \delta(w)}{\int_{0}^{R} d u \exp \left[-\frac{k v_{0}}{f_{0} D_{v}} \sin \frac{\theta}{2}\left(u-u_{0}\right)^{2}\right]} .
$$

However, this approximation stops being valid when the angle becomes small enough that $u_{0}>R$. This means that the torque and the potential [Fig. 4(b)] still have to be evaluated numerically.

\section{Bundle stability}

The bundling and unbundling probabilities are related to the generalized potential as $P_{B, U}=\mathcal{N} \int_{\Theta_{B, U}} e^{-U(\theta)} d \theta$, where the normalization factor reads $\mathcal{N}=1 / \int_{0}^{\pi} e^{U(\theta)} d \theta$. For angles $\theta>\theta_{\max }$, the potential contribution from cross-linking proteins is negligible, so we can write

$$
P_{U}=\mathcal{N} \int_{\theta_{\max }}^{\pi} \sin \theta d \theta
$$


Note that if the interaction between the MTs is attractive for all angles and the potential barrier between bundled and unbundled state does not exist, we automatically consider the bundles stable. The bundling probability is calculated using the small angle approximation:

$$
P_{B}=\mathcal{N}\left[\exp \left(\frac{\theta_{\max }}{\theta_{\min }}\right) \int_{0}^{\theta_{\min }} \theta d \theta+\int_{\theta_{\min }}^{\theta_{\max }} \exp \left(\frac{\theta_{\max }}{\theta}\right) \theta d \theta\right] .
$$

The integrals in Eqs. (A23) and (A24) can be evaluated analytically for $R \gg y_{0}$, and after using the definitions for $\theta_{\max }$ and $\theta_{\min }$, we obtain

$$
P_{B}=1-\frac{1}{1+\left(\frac{y_{0}}{2 R}\right)^{2} \exp \left(\frac{k_{a} c_{0}}{k_{d 0}} y_{0} \pi\right)} .
$$

The stability condition, Eq. (11), can be written explicitly as

$$
\left(\frac{y_{0}}{2 R}\right)^{2} \exp \left(\frac{k_{a} c_{0}}{k_{d 0}} y_{0} \pi\right)>1 \text {. }
$$

For plus-end directed motors, we use the approximation in Eq. (A22) to numerically determine the region of stability.

To calculate the average bundling time, we start by writing the equation for the average first passage time from some angle $\theta$ to the minimum of the potential, $\theta_{\min }[46]$ :

$$
\langle t\rangle_{\theta, \theta_{\min }}=\frac{1}{D} \int_{\theta_{\min }}^{\theta} d \theta^{\prime} \int_{0}^{\theta^{\prime}} \frac{\int_{\theta^{\prime}}^{\pi} p_{\theta}\left(\theta^{\prime \prime}\right) d \theta^{\prime \prime}}{p_{\theta}\left(\theta^{\prime}\right)} .
$$

We assume that MTs are "introduced into the system" at a random, isotropically distributed starting angle in the region $\Theta_{U}$, so the average bundling time is obtained by taking the average of the first passage time over all initial angles, $\left\langle t_{B}\right\rangle=\frac{1}{2} \int_{\theta_{\min }}^{\pi}\langle t\rangle_{\theta, \theta_{\min }} \sin \theta d \theta$. The average unbundling time is calculated as $\left\langle t_{\mathrm{U}}\right\rangle=\frac{1}{2} \int_{\theta_{\min }}^{\pi} d \theta \sin \theta\langle t\rangle_{\theta_{\min }, \theta}$, where the first passage time from $\theta_{\min }$ to $\theta,\langle t\rangle_{\theta_{\min }, \theta}$, is calculated analogously to Eq. (A27). The evaluation of the triple integrals required for obtaining the average bundling and unbundling time is done numerically.

\section{Numerical simulations of $\theta(t)$}

The equation of motion for $\theta(t)$ is given explicitly in Eq. (9), which is a first-order stochastic differential equation. To produce the time courses shown in Fig. 2, we opted for an Euler-Maruyama scheme with an adaptive time-step, which is constant for all angles larger than some limit angle and is inversely proportional to the angle when it is smaller. This is because the value of the deterministic term changes rapidly between time steps when the angles are close to zero, but varies slowly at larger angles, so using a higher order convergence scheme would be computationally inefficient. Finally, to ensure numerical stability, we also imposed a reflective boundary condition at $\theta=0$. Full Matlab code is available on request.

\section{Choice of parameter values}

Our model has 10 constant parameters, which are estimated based on previous measurements (see Table I). The rest lengths of passive crosslinkers, $y_{0}=40 \mathrm{~nm}$, and motors, $y_{0, m}=60 \mathrm{~nm}$, are estimated from EM data for PRC1 [42,53] and Kinesin-5 [54], respectively. The spring stiffness of both passive crosslinkers and motors is taken to be $k=100 \mathrm{pN} / \mu \mathrm{m}$, which corresponds to the value for $\mathrm{Eg} 5$ measured in Ref. [55] and used in Ref. [14], while being three times lower than the value measured in Ref. [56]. Note that the stiffness of PRC1/Ase1 is not measured, but it is presumably higher than the stiffness of Eg5 due to the difference in length. The values for attachment and detachment rates, $k_{\text {on }}=$ $0.01 \mathrm{~s}^{-1}$ and $k_{\text {off }}=0.1 \mathrm{~s}^{-1}$, are taken from measurements on Ase1 [13], and the detachment rate is similar to the one estimated from the dwell time of Cin8 [57]. Detachment is additionally described by the critical force required to rupture the bond between the cross-linking protein and the MT, $f_{\mathrm{c}}$, for which we used the value measured for kinesin-1 [58]. Passive crosslinkers perform Brownian motion along the MTs with the diffusion constant $D_{0}$, whose value is taken from measurements for Ase1 [13,41]. The movement of motors is described by their velocity at zero load, $v_{0}$, which is estimated in vitro motility assays for Cut7 [59], with the stall force, $f_{0}$, for which we used the value measured for Cin8 from budding yeast [60], consistent with the stall force estimated for Xenopus kinesin-5 [61]. Finally, the MTs are characterized by their diffusion constant, $D$, which is calculated, according to the theory for angular diffusion of rigid rods [62] as $D \propto$ $R^{-3}$, using fitting results from [26], which are consistent with our own measurements for two different values of MT length.

\section{APPENDIX B: EXPERIMENTAL METHODS}

\section{Strains and media}

Fission yeast strains used in this study are listed in Supplemental Material Table I [38]. The KI061 (cdc25-22 ndc80nmtP41-tdTomato-kanMX6 kanr-nmtP3-GFP-atb2+ sid4GFP-kanr, created by Iana Kalinina, Tolić lab, Max Planck Institute of Molecular Cell Biology and Genetics, Dresden) was used as the parent strain for deletion strains. The strains were obtained by crossing and random spore analysis [63]. The ase1::hygR ura4-D18 leu1-32 strain, used to create LW050, was a kind gift from Prof. Jonathan Millar [64]. All the strains were grown on Yeast Extract (YE) medium agar plates with appropriate supplements (ade, leu, ura, his, lys) and $2 \mu \mathrm{M}$ thiamine in incubator (Heraeus B6) at $25^{\circ} \mathrm{C}$.

\section{Culture dish customization}

Culture dish (MatTek Corporation, $35 \mathrm{~mm}$ ) wall was cut to $2 \mathrm{~mm}$ height. Original cover slip was removed from the dish bottom and the remaining culture dish was soaked in $70 \%$ ethanol. Cover slips (Sigma-Aldrich, Corning, $22 \mathrm{~mm} \times$ $22 \mathrm{~mm}$ ) were washed in 2-propanol and attached to the prewashed culture dish with nail polish. Prepared dishes were used for microscopy.

\section{Sample preparation}

The strains were grown on Yeast Extract medium agar plates with appropriate supplements (YES) and $2 \mu \mathrm{M}$ thiamine in incubator (Heraeus B6) at $25^{\circ} \mathrm{C}$ overnight. A 
loopful of grown cells was cultured in liquid YES with thiamine in a shaking incubator (ISF-1-W, Kuhner Shaker) at $25^{\circ} \mathrm{C}$ for $2-3$ hours to induce mitosis. Prepared dish was coated with lectin (Sigma-Aldrich) $30 \mathrm{~min}$ prior to usage to ensure strong attachment of cells. $200 \mu$ l of liquid culture was placed on pretreated culture dish for $25 \mathrm{~min}$ for sedimentation. The cells were washed two times with $200 \mu$ l of YES with thiamine, followed by final $200 \mu \mathrm{l}$ of YES with thiamine and closing the dish on top with a cover slip (Corning) to keep the cells from drying out.

\section{Microtubule depolymerization by cold treatment}

Custom-made fast thermoelectric device that can be coupled to the microscope was used to depolymerize and repolymerize microtubules by shifting the temperature. To induce depolymerization, temperature was kept at $0{ }^{\circ} \mathrm{C}$ for $15 \mathrm{~min}$ to ensure that all the microtubules from mitotic spindles were disassembled. Subsequent re-polymerization was induced by raising the temperature to $24^{\circ} \mathrm{C}$.

\section{Time-lapse live-cell imaging}

Live-cell imaging was preformed using an Andor Revolution Spinning Disk System (Andor Technology), consisting of a Yokogawa CSU-X1 (10.000 rpm) spinning-disc scan head (Yokogawa Electric Corporation) with a 405/488/561 nm Yokogawa dichroic beam splitter (Semrock). The scan head was connected to an Olympus IX71 microscope (Olympus) with an inverted stand, equipped with a fast piezo objective zpositioner (PIFOC, Physik Instrumente GmbH \& K.G.), Prior ProScanIII xy scanning stage and an Olympus UPlanSApo $\times$ 100/1.4 NA oil objective (Olympus). For excitation, a sapphire $488 \mathrm{~nm}$ solid-state laser (75 mW, Coherent) was used with the laser intensity set to $6 \%$ using the acousto-optic tunable filter (ALC, Andor Technology). Emission wavelength was selected using the respective emission filter 525/30. Andor iXon EM+ DU-897 BV back illuminated EMCCD with pixel size of EMCCD chip $16 \mu \mathrm{m}$ (Andor Technology), cooled to $-80^{\circ} \mathrm{C}$, electron multiplication gain 300 was used for acquisition. Three-dimensional time-lapse stacks comprising 13 optical sections at $0.5 \mu \mathrm{m} \mathrm{z}$-spacing were taken every $2 \mathrm{~s}$ with exposure times 0.08 and $0.06 \mathrm{~s}$. The $\mathrm{x}-\mathrm{y}$ pixel size in the images was $168 \mathrm{~nm}$. Andor iQ software version 2.9 (Andor Technology) was used to control the system.

Cells expressing Mal3-GFP and Sid4-GFP were imaged using Bruker Opterra Multipoint Scanning Confocal Microscope (Bruker Nano Surfaces, Middleton, WI). The system was mounted on a Nikon Ti-E inverted microscope equipped with a Nikon CFI Plan Apo VC 100x/1.4 numerical aperture oil objective (Nikon, Tokyo, Japan). During imaging, cells were maintained at $25^{\circ} \mathrm{C}$ in Okolab Cage Incubator (Okolab, Pozzuoli, NA, Italy). For excitation of GFP fluorescence, a $488 \mathrm{~nm}$ diode laser line was used. The excitation light was separated from the emitted fluorescence using Opterra Dichroic and Barrier Filter Set 405/488/561/640. Images were captured with an Evolve 512 Delta EMCCD Camera (Photometrics, Tucson, AZ) using $50 \mathrm{~ms}$ exposure times. To bring the xy-pixel size in the image down to $83 \mathrm{~nm}$, a 2x relay lens was placed in front of the camera. Z-stacks were acquired comprising five focal planes at a $0.5 \mu \mathrm{m}$ z-spacing. Image acquisition was performed for 300 time frames at $0.75 \mathrm{~s}$ intervals. The system was controlled with the Prairie View Imaging Software (Bruker).

\section{Image analysis}

Image processing was performed in ImageJ (National Institutes of Health, Bethesda, MD). Quantification and statistical analysis were done in MatLab (MathWorks, Natick, MA). Spindle poles and plus ends of MTs in cells expressing tubulin-GFP were tracked manually in ImageJ. Tracking in cells expressing Mal3-GFP and Sid4-GFP was preformed automatically using Low Light Tracking Tool, an ImageJ plugin [65]. Tracking was performed on maximum-intensity projections of the z-stacks. Bundling events were confirmed by the increase in the signal intensity of a cross-section of the spindle. The signal intensity was measured in ImageJ by drawing 3-pixel-thick line perpendicular to the spindle. Bundling time was calculated as the total observation time of MTs divided by the number of bundling events in which MT bundled into the spindle.
[1] N. Pavin and I. M. Tolic, Self-organization and forces in the mitotic spindle, Annu. Rev. Biophys. 45, 279 (2016).

[2] M. Kirschner and T. Mitchison, Beyond self-assembly: From microtubules to morphogenesis, Cell 45, 329 (1986).

[3] K. L. McDonald, E. T. O'Toole, D. N. Mastronarde, and J. R. McIntosh, Kinetochore microtubules in PTK cells, J. Cell Biol. 118, 369 (1992).

[4] D. N. Mastronarde, K. L. McDonald, R. Ding, and J. R. McIntosh, Interpolar spindle microtubules in PTK cells, J. Cell Biol. 123, 1475 (1993).

[5] R. Ding, K. L. McDonald, and J. R. McIntosh, Threedimensional reconstruction and analysis of mitotic spindles from the yeast, Schizosaccharomyces pombe, J. Cell Biol. 120, 141 (1993).

[6] D. Pellman, M. Bagget, Y. Tu, G. R. Fink, and H. Tu, Two microtubule-associated proteins required for anaphase spindle movement in Saccharomyces cerevisiae, J. Cell Biol. 130, 1373 (1995).

[7] I. Hagen and M. Yanagida, Novel potential mitotic motor protein encoded by the fission yeast cut $7^{+}$gene, Nature 347, 563 (1990).

[8] R. Le Guellec, J. Paris, A. Couturier, C. Roghi, and M. Philippe, Cloning by differential screening of a Xenopus cDNA that encodes a kinesin-related protein, Mol. Cell. Biol. 11, 3395 (1991).

[9] S. A. Endow, S. Henikoff, and L. Soler-Niedziela, Mediation of meiotic and early mitotic chromosome segregation in Drosophila by a protein related to kinesin, Nature 345, 81 (1990).

[10] H. B. McDonald, R. J. Stewart, and L. S. Goldstein, The kinesin-like ncd protein of Drosophila is a minus end-directed microtubule motor, Cell 63, 1159 (1990). 
[11] F. Nédélec, Computer simulations reveal motor properties generating stable antiparallel microtubule interactions, J. Cell Biol. 158, 1005 (2002).

[12] M. Braun, Z. Lansky, G. Fink, F. Ruhnow, S. Diez, and M. E. Janson, Adaptive braking by Ase1 prevents overlapping microtubules from sliding completely apart, Nat. Cell Biol. 13, 1259 (2011).

[13] Z. Lansky, M. Braun, A. Ludecke, M. Schlierf, P. R. ten Wolde, M. E. Janson, and S. Diez, Diffusible crosslinkers generate directed forces in microtubule networks, Cell 160, 1159 (2015).

[14] D. Johann, D. Goswami, and K. Kruse, Generation of Stable Overlaps between Antiparallel Filaments, Phys. Rev. Lett. 115, 118103 (2015).

[15] E. N. Cytrynbaum, J. M. Scholey, and A. Mogilner, A force balance model of early spindle pole separation in Drosophila embryos, Biophys. J. 84, 757 (2003).

[16] E. N. Cytrynbaum, P. Sommi, I. Brust-Mascher, J. M. Scholey, and A. Mogilner, Early spindle assembly in Drosophila embryos: Role of a force balance involving cytoskeletal dynamics and nuclear mechanics, Mol. Biol. Cell 16, 4967 (2005).

[17] R. Wollman, G. Civelekoglu-Scholey, J. M. Scholey, and A. Mogilner, Reverse engineering of force integration during mitosis in the Drosophila embryo, Mol. Syst. Biol. 4, 195 (2008).

[18] K. S. Burbank, T. J. Mitchison, and D. S. Fisher, Slide-andcluster models for spindle assembly, Curr. Biol. 17, 1373 (2007).

[19] R. Loughlin, R. Heald, and F. Nedelec, A computational model predicts Xenopus meiotic spindle organization, J. Cell Biol. 191, 1239 (2010).

[20] S. B. Reber, J. Baumgart, P. O. Widlund, A. Pozniakovsky, J. Howard, A. A. Hyman, and F. Julicher, XMAP215 activity sets spindle length by controlling the total mass of spindle microtubules, Nat. Cell Biol. 15, 1116 (2013).

[21] J. Brugues and D. Needleman, Physical basis of spindle selforganization, Proc. Natl. Acad. Sci. USA 111, 18496 (2014).

[22] A. J. Hepperla et al., Minus-end-directed kinesin-14 motors align antiparallel microtubules to control metaphase spindle length, Dev. Cell 31, 61 (2014).

[23] L. Winters, I. Ban, M. Prelogovic, I. Kalinina, N. Pavin, and I. M. Tolic, Pivoting of microtubules driven by minus-enddirected motors leads to spindle assembly, BMC Biol. 17, 42 (2019).

[24] S. Baumgartner and I. M. Tolic, Astral microtubule pivoting promotes their search for cortical anchor sites during mitosis in budding yeast, PLoS One 9, e93781 (2014).

[25] R. Blackwell et al., Physical determinants of bipolar mitotic spindle assembly and stability in fission yeast, Sci. Adv. 3, e1601603 (2017).

[26] I. Kalinina et al., Pivoting of microtubules around the spindle pole accelerates kinetochore capture, Nat. Cell Biol. 15, 82 (2013).

[27] R. Blackwell et al., Contributions of microtubule dynamic instability and rotational diffusion to kinetochore capture, Biophys. J. 112, 552 (2017).

[28] G. Cojoc, A. M. Florescu, A. Krull, A. H. Klemm, N. Pavin, F. Julicher, and I. M. Tolic, Paired arrangement of kinetochores together with microtubule pivoting and dynamics drive kinetochore capture in meiosis I, Sci. Rep. 6, 25736 (2016).
[29] N. Pavin and I. M. Tolic-Norrelykke, Swinging a sword: How microtubules search for their targets, Syst. Synth. Biol. 8, 179 (2014).

[30] A. R. Lamson, C. J. Edelmaier, M. A. Glaser, and M. D. Betterton, Theory of cytoskeletal reorganization during crosslinker-mediated mitotic spindle assembly, Biophys. J. 116, 1719 (2019).

[31] S. A. Rincon, A. Lamson, R. Blackwell, V. Syrovatkina, V. Fraisier, A. Paoletti, M. D. Betterton, and P. T. Tran, Kinesin-5independent mitotic spindle assembly requires the antiparallel microtubule crosslinker Ase1 in fission yeast, Nat. Commun. 8, 15286 (2017).

[32] C. J. Edelmaier, A. R. Lamson, Z. R. Gergely, S. Ansari, R. Blackwell, J. R. McIntosh, M. A. Glaser, and M. D. Betterton, Mechanisms of chromosome biorientation and bipolar spindle assembly analyzed by computational modeling, bioRxiv 649913 (2019), doi: 10.1101/649913.

[33] F. Ziebert, M. Vershinin, S. P. Gross, and I. S. Aranson, Collective alignment of polar filaments by molecular motors, Eur. Phys. J. E Soft Matter 28, 401 (2009).

[34] S. K. Vogel, N. Pavin, N. Maghelli, F. Julicher, and I. M. Tolic-Norrelykke, Self-organization of dynein motors generates meiotic nuclear oscillations, PLoS Biol. 7, e1000087 (2009).

[35] A. Yamamoto, R. R. West, J. R. McIntosh, and Y. Hiraoka, A cytoplasmic dynein heavy chain is required for oscillatory nuclear movement of meiotic prophase and efficient meiotic recombination in fission yeast, J. Cell Biol. 145, 1233 (1999).

[36] A. Yamashita, M. Sato, A. Fujita, M. Yamamoto, and T. Toda, The roles of fission yeast ase1 in mitotic cell division, meiotic nuclear oscillation, and cytokinesis checkpoint signaling, Mol. Biol. Cell 16, 1378 (2005).

[37] Y. Gachet, C. Reyes, T. Courtheoux, S. Goldstone, G. Gay, C. Serrurier, and S. Tournier, Sister kinetochore recapture in fission yeast occurs by two distinct mechanisms, both requiring Dam1 and Klp2, Mol. Biol. Cell. 19, 1646 (2008).

[38] See Supplemental Material at http://link.aps.org/supplemental/ 10.1103/PhysRevE.100.012403 for supplementary movies, figures, and tables.

[39] J. D. Beinhauer, I. M. Hagan, J. H. Hegemann, and U. Fleig, Mal3, the fission yeast homologue of the human APCinteracting protein EB-1 is required for microtubule integrity and the maintenance of cell form, J. Cell Biol. 139, 717 (1997).

[40] I. Loiodice, J. Staub, T. G. Setty, N. P. Nguyen, A. Paoletti, and P. T. Tran, Ase1p organizes antiparallel microtubule arrays during interphase and mitosis in fission yeast, Mol. Biol. Cell 16, 1756 (2005).

[41] L. C. Kapitein, M. E. Janson, S. M. van den Wildenberg, C. C. Hoogenraad, C. F. Schmidt, and E. J. Peterman, Microtubuledriven multimerization recruits ase $1 \mathrm{p}$ onto overlapping microtubules, Curr. Biol. 18, 1713 (2008).

[42] R. Subramanian, E. M. Wilson-Kubalek, C. P. Arthur, M. J. Bick, E. A. Campbell, S. A. Darst, R. A. Milligan, and T. M. Kapoor, Insights into antiparallel microtubule crosslinking by PRC1, a conserved nonmotor microtubule binding protein, Cell 142, 433 (2010).

[43] P. Bieling, I. A. Telley, and T. Surrey, A minimal midzone protein module controls formation and length of antiparallel microtubule overlaps, Cell 142, 420 (2010).

[44] C. Fu, J. J. Ward, I. Loiodice, G. Velve-Casquillas, F. J. Nedelec, and P. T. Tran, Phospho-regulated interaction between kinesin-6 
Klp9p and microtubule bundler Ase1p promotes spindle elongation, Dev. Cell 17, 257 (2009).

[45] H. A. Kramers, Brownian motion in a field of force and the diffusion model of chemical reactions, Physica 7, 284 (1940).

[46] C. W. Gardiner, Handbook of Stochastic Methods (Springer, Berlin, 1985), Vol. 4.

[47] J. J. Ward, H. Roque, C. Antony, and F. Nedelec, Mechanical design principles of a mitotic spindle, Elife 3, e03398 (2014).

[48] T. Murata, S. Sonobe, T. I. Baskin, S. Hyodo, S. Hasezawa, T. Nagata, T. Horio, and M. Hasebe, Microtubule-dependent microtubule nucleation based on recruitment of gamma-tubulin in higher plants, Nat. Cell Biol. 7, 961 (2005).

[49] G. Goshima, M. Mayer, N. Zhang, N. Stuurman, and R. D. Vale, Augmin: A protein complex required for centrosomeindependent microtubule generation within the spindle, J. Cell Biol. 181, 421 (2008).

[50] N. M. Mahoney, G. Goshima, A. D. Douglass, and R. D. Vale, Making microtubules and mitotic spindles in cells without functional centrosomes, Curr. Biol. 16, 564 (2006).

[51] S. Petry, A. C. Groen, K. Ishihara, T. J. Mitchison, and R. D. Vale, Branching microtubule nucleation in Xenopus egg extracts mediated by augmin and TPX2, Cell 152, 768 (2013).

[52] E. P. Hsu, Stochastic Analysis on Manifolds (American Mathematical Society, Providence, RI, 2002), Vol. 38.

[53] E. H. Kellogg, S. Howes, S. C. Ti, E. Ramirez-Aportela, T. M. Kapoor, P. Chacon, and E. Nogales, Near-atomic cryo-EM structure of PRC1 bound to the microtubule, Proc. Natl. Acad. Sci. USA 113, 9430 (2016).

[54] A. S. Kashina, R. J. Baskin, D. G. Cole, K. P. Wedaman, W. M. Saxton, and J. M. Scholey, A bipolar kinesin, Nature 379, 270 (1996).
[55] M. T. Valentine, P. M. Fordyce, T. C. Krzysiak, S. P. Gilbert, and S. M. Block, Individual dimers of the mitotic kinesin motor Eg5 step processively and support substantial loads in vitro, Nat. Cell Biol. 8, 470 (2006).

[56] K. Kawaguchi and S. Ishiwata, Nucleotide-dependent singleto double-headed binding of kinesin, Science 291, 667 (2001).

[57] J. Roostalu, C. Hentrich, P. Bieling, I. A. Telley, E. Schiebel, and T. Surrey, Directional switching of the kinesin Cin8 through motor coupling, Science 332, 94 (2011).

[58] M. J. Schnitzer, K. Visscher, and S. M. Block, Force production by single kinesin motors, Nat. Cell Biol. 2, 718 (2000).

[59] M. Edamatsu, Bidirectional motility of the fission yeast kinesin5, Cut7, Biochem. Biophys. Res. Commun. 446, 231 (2014).

[60] T. Fallesen, J. Roostalu, C. Duellberg, G. Pruessner, and T. Surrey, Ensembles of bidirectional kinesin Cin8 produce additive forces in both directions of movement, Biophys. J. 113, 2055 (2017).

[61] Y. Shimamoto, S. Forth, and T. M. Kapoor, Measuring pushing and braking forces generated by ensembles of kinesin-5 crosslinking two microtubules, Dev. Cell 34, 669 (2015).

[62] M. M. Tirado and J. G. de la Torre, Translational friction coefficients of rigid, symmetric top macromolecules. Application to circular cylinders, J. Chem. Phys. 71, 2581 (1979).

[63] http://www-bcf.usc.edu/ forsburg/spores.html.

[64] J. C. Meadows and J. Millar, Latrunculin A delays anaphase onset in fission yeast by disrupting an Ase1-independent pathway controlling mitotic spindle stability, Mol. Biol. Cell 19, 3713 (2008).

[65] A. Krull, A. Steinborn, V. Ananthanarayanan, D. RamunnoJohnson, U. Petersohn, and I. M. Tolic-Norrelykke, A divide and conquer strategy for the maximum likelihood localization of low intensity objects, Opt. Express 22, 210 (2014). 